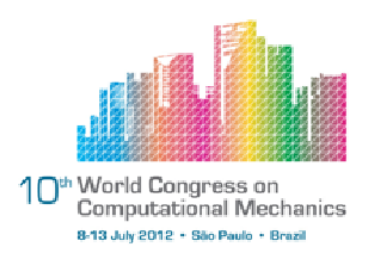

\title{
MATERIAL PARAMETER IDENTIFICATION ON METAL MATRIX COMPOSITES
}

\author{
G. J. Jansen van Rensburg ${ }^{1,2}$, S. Kok ${ }^{1}$, D. N. Wilke ${ }^{2}$ \\ ${ }^{1}$ Modelling and Digital Science, The Council for Scientific and Industrial Research, South \\ Africa (jjvrensburg@csir.co.za) \\ ${ }^{2}$ Department of Mechanical and Aeronautical Engineering, University of Pretoria
}

\begin{abstract}
Tests were done on the compressive behaviour of different metal matrix composite materials. These extremely hard engineering materials consist of ceramic particles embedded in a metal alloy binder. Due to the high stiffness and brittle nature of the material, compression tests were only performed to about $2 \%$ uniaxial strain as measured by the displacement of the hydraulic cylinder. In room temperature tests, three strain gauges are secured evenly around the centre of the test section. The results from these strain gauges indicate that some compression instability, eccentric loading or other resulting bending condition is present.

In this work, a finite element inverse analysis is employed to determine not only material parameters but also the boundary conditions that best replicate the experimental data. The quality of the fits is subject to the limits of the material model and boundary parameterisation. An alternative procedure that uses the time and strain history to evolve the yield stress is also employed to approximate the material parameters. The Mechanical Threshold Stress model is used to model the materials.
\end{abstract}

Keywords: Inverse analysis, Material parameter identification, Mechanical Threshold Stress, Metal Matrix Composite.

\section{INTRODUCTION}

The Mechanical Threshold Stress (MTS) model [4] has demonstrated the ability to accurately model the effect of temperature and plastic strain rate on the post yielding behaviour of metals. In this work, the ability of the model to capture the post yielding behaviour of Metal Matrix Composites (MMCs) is inspected. The MTS material parameters are identified by a finite element based inverse analysis that aims to also determine experimental conditions, as well as lesser computationally expensive techniques.

The specific MMC materials investigated are to be used in processes where high temperatures and pressures are present. In these processes it has been observed that the material undergoes plastic deformation. The accurate modelling of the elastic-viscoplastic behaviour of the material has to be taken into account during the modelling of the process, due to the 
extreme working conditions under which these materials are used. The experimental data is obtained from compression tests with the data from 3 strain gauges available at room temperature, along with the hydraulic cylinder displacement and load cell data. The strain gauges are fixed to the centre of the experimental test specimen, $120^{\circ}$ apart. Strain data from a single extensometer is also available at $150^{\circ} \mathrm{C}, 250^{\circ} \mathrm{C}, 350^{\circ} \mathrm{C}$ and $500^{\circ} \mathrm{C}$.

\subsection{The Mechanical Threshold Stress model}

In the MTS model formulation, the flow stress $\sigma_{y}$ of a material is developed by scaling the mechanical threshold to account for rate and temperature dependence [4]. The mechanical threshold, $\hat{\sigma}$, is defined as the flow stress at $0 \mathrm{~K}$ and is separated into an athermal $\hat{\sigma}_{a}$ and thermal component $\hat{\sigma}_{t}^{\kappa}$. Here, $\hat{\sigma}_{a}$ characterizes the rate-independent interactions of dislocations with long-range barriers, while $\hat{\sigma}_{t}^{\kappa}$ characterizes the rate-dependent interactions of dislocations with short-range obstacles that can be overcome with the assistance of thermal activation [4].

At different temperatures $T$ and plastic strain rates $\dot{\varepsilon}$, the contributions to the flow stress $\sigma_{t}^{\kappa}$ are related to their reference counterparts $\hat{\sigma}_{t}^{\kappa}$ through the scaling functions $S_{t}^{\kappa}(\dot{\varepsilon}, T)$, so that $\sigma_{t}^{\kappa}=\hat{\sigma}_{t}^{\kappa} S_{t}^{\kappa}(\dot{\varepsilon}, T)$. This implies that the flow stress of a material, $\sigma_{y}$, is expressed as

$$
\frac{\sigma_{y}}{\mu}=\frac{\hat{\sigma}_{a}}{\mu}+\sum_{\kappa} \frac{\sigma_{t}^{\kappa}}{\mu}=\frac{\hat{\sigma}_{a}}{\mu}+\sum_{\kappa} S_{t}^{\kappa}(\dot{\varepsilon}, T) \frac{\hat{\sigma}_{t}^{\kappa}}{\mu_{o}} .
$$

Here, $\mu_{o}$ is a reference value of the shear modulus $\mu$, which is modeled by [9]

$$
\mu=\tilde{\mu}(T)=\mu_{o}-\frac{D_{o}}{\exp \left(\frac{T_{o}}{T}\right)-1}
$$

with empirical constants $T_{o}$ and $D_{o}$. The temperature dependence of $\mu$ is included in the scaling functions $S_{t}^{\kappa}$. The material stiffness is also coupled to these constants through the shear modulus by

$$
E=2 \mu(1+\nu)
$$

where $\nu$ is chosen as 0.21 .

If an Arrhenius expression is used to describe interaction kinetics for short-range obstacles, and a phenomenological relation is used for the free energy function of stress [5], each thermal component of the flow stress $\left(\sigma_{t}^{\kappa}\right)$ is expressed as a function of the thermal component of the mechanical threshold $\left(\hat{\sigma}_{t}^{\kappa}\right)$ by

$$
\frac{\sigma_{t}^{\kappa}}{\mu}=S_{t}^{\kappa}(\dot{\varepsilon}, T) \frac{\hat{\sigma}_{t}^{\kappa}}{\mu_{o}}=\left[1-\left(\frac{k T}{g_{o} \mu b^{3}} \ln \frac{\dot{\varepsilon}_{o}}{\dot{\varepsilon}}\right)^{1 / q}\right]^{1 / p} \frac{\hat{\sigma}_{t}^{\kappa}}{\mu_{o}} .
$$


Here $k$ is the Boltzmann constant, $b$ is the magnitude of the Burger's vector, $g_{o}$ is the normalized activation energy for dislocations to overcome the obstacles, $\dot{\varepsilon}_{o}$ is a constant and $p$ and $q$ are statistical constants that characterize the shape of the obstacle profile $(0 \leq p \leq 1,1 \leq$ $q \leq 2)[5]$.

In the standard MTS model, there are two thermal components, i.e. $\hat{\sigma}_{t}^{\kappa}, \kappa=1,2$. Using the notation $\hat{\sigma}_{t}^{1}=\hat{\sigma}_{i}$ and $\hat{\sigma}_{t}^{2}=\hat{\sigma}_{\varepsilon}$, Eq.(1) is written as

$$
\frac{\sigma_{y}}{\mu}=\frac{\sigma_{a}}{\mu}+S_{i}(\dot{\varepsilon}, T) \frac{\hat{\sigma}_{i}}{\mu_{o}}+S_{\varepsilon}(\dot{\varepsilon}, T) \frac{\hat{\sigma}_{\varepsilon}}{\mu_{o}},
$$

where

$$
S_{i}=\left[1-\left(\frac{k T}{g_{o i} \mu b^{3}} \ln \frac{\dot{\varepsilon}_{o i}}{\dot{\varepsilon}}\right)^{1 / q_{i}}\right]^{1 / p_{i}} \quad S_{\varepsilon}=\left[1-\left(\frac{k T}{g_{o \varepsilon} \mu b^{3}} \ln \frac{\dot{\varepsilon}_{o \varepsilon}}{\dot{\varepsilon}}\right)^{1 / q_{\varepsilon}}\right]^{1 / p_{\varepsilon}} .
$$

Note that $\hat{\sigma}_{i}$ describes the thermal portion of the yield stress (which does not evolve), while $\hat{\sigma}_{\varepsilon}$ describes the interaction of mobile dislocations with the forest dislocation structure (which does evolve).

The evolution of $\hat{\sigma}_{\varepsilon}$ is given in rate form, by

$$
\frac{d \hat{\sigma}_{\varepsilon}}{d \varepsilon}=\theta\left(T, \dot{\varepsilon}, \hat{\sigma}_{\varepsilon}\right)=\theta_{o}-\theta_{r}\left(T, \dot{\varepsilon}, \hat{\sigma}_{\varepsilon}\right)
$$

where $\theta_{o}$ is the hardening due to dislocation accumulation (assumed constant) and $\theta_{r}$ is the dynamic recovery rate. The functional form of the hardening rate $\theta$ is chosen to fit experimental data, and popular choices include a tanh form [2, 4] and a power law form [6], given by

$$
\theta=\theta_{o}\left(1-\frac{\tanh \left[\frac{\alpha \hat{\sigma}_{\varepsilon}^{s}}{\hat{\sigma}_{\varepsilon s}}\right]}{\tanh (\alpha)}\right) \text { and } \theta=\theta_{o}\left[1-\frac{\hat{\sigma}_{\varepsilon}^{s}}{\hat{\sigma}_{\varepsilon s}}\right]^{\kappa}
$$

Both $\alpha$ and $\kappa$ are fitted constants while $\hat{\sigma}_{\varepsilon s}$ is the saturation threshold stress. In both of these hardening laws, $\theta_{o}$ assumes the role of the initial hardening rate. For this work, the tanh hardening form is chosen with $\alpha=2$ [4].

The saturation threshold stress $\hat{\sigma}_{\varepsilon s}$ is a function of both strain rate and temperature, through the relation [2]

$$
\ln \frac{\dot{\varepsilon}}{\dot{\varepsilon}_{\varepsilon s o}}=\frac{g_{o \varepsilon s} \mu b^{3}}{k T} \ln \frac{\hat{\sigma}_{\varepsilon s}}{\hat{\sigma}_{\varepsilon s o}}
$$

where $\dot{\varepsilon}_{\varepsilon s o}, g_{o \varepsilon s}$ and $\hat{\sigma}_{\varepsilon s o}$ are empirically obtained constants. 


\subsection{Experimental Data}

The materials tested are used in high temperature and pressure applications. Specifics regarding application, exact material compound and grade is proprietary information, therefore all figure axes and material parameter values are normalized in this report. A material test specimen similar to the one described by [3] is used for the experimental compression tests. This compression test specimen is basically a modified version of a conventional cylindrical tensile specimen where the length of the test section is reduced to avoid buckling and shear deformation modes during the compression test.

One of the demanding aspects of compression tests on these hard materials is a very high compressive strength and stiffness. The testing machine stiffness, which should ideally be orders of magnitude greater than that of the specimen, is inadequate in this case. Experimental data indicates that for a typical test the hydraulic cylinder displaces $1.4 \mathrm{~mm}$, while the test specimen only decreases in height by about $0.4 \mathrm{~mm}$. The remaining $1.0 \mathrm{~mm}$ displacement occurs in elastic displacement of the testing machine frame.

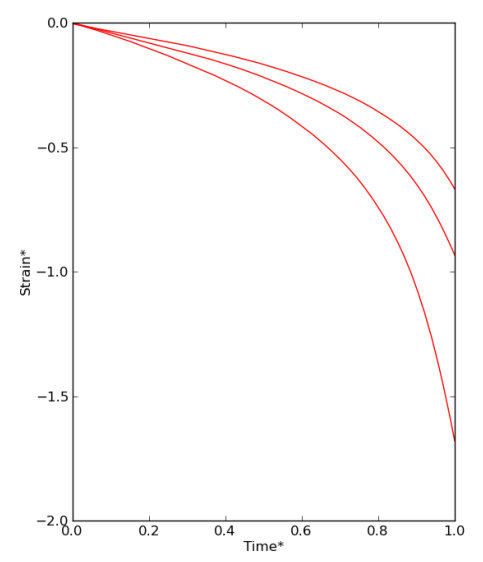

(a)

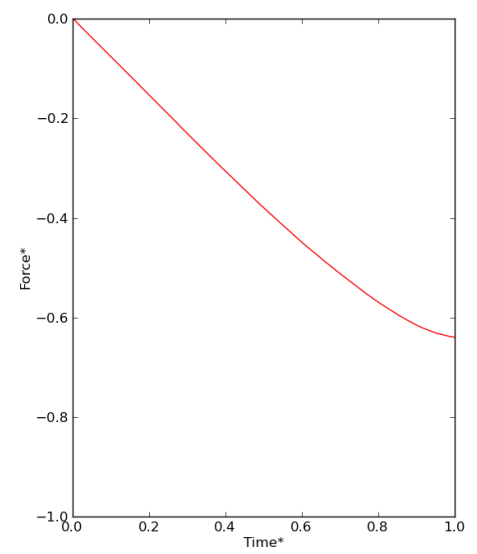

(d)

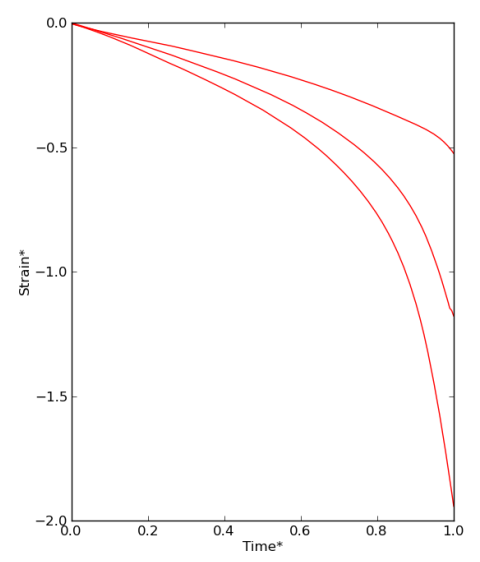

(b)

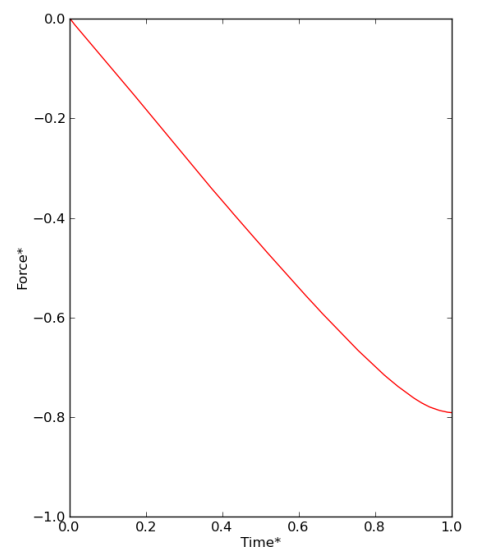

(e)

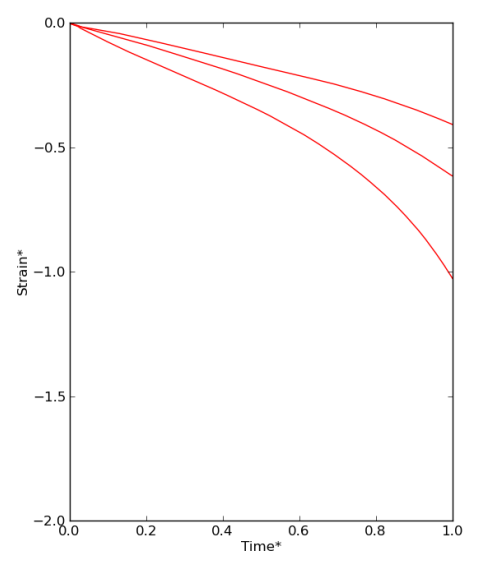

(c)

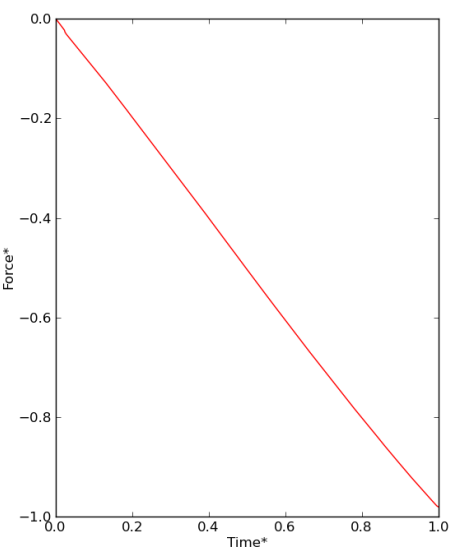

(f)

Figure 1. Normalised experimental strains and forces of three materials at room temperature. (a), (b) and (c) show the strains recorded by the strain gauges spaced evenly around the circumferance of the test section while (d), (e) and (f) show the load cell data for the same tests. All of the axes are normalized with the same factor. 
From the strain gauge data of the room temperature tests, it appears that there is some compressive instability, eccentric load condition or equivalent bending moment. This can be seen in the strain gauge data over time of three distinct tests on different materials in Figures 1(a), (b) and (c). The corresponding load cell data recorded over time is visible in Figures 1(d), (e) and (f). Unfortunately data from only a single extensometer is available for the elevated temperature tests. From the room temperature tests it seems plausible that the elevated temperature tests would also experience a similar non-ideal loading condition, but this cannot be taken into account due to the data limitation.

\section{INVERSE ANALYSIS}

\subsection{Finite Element Based}

The MTS material parameters are determined by an inverse analysis on a finite element simulation. The material parameters and boundary conditions are sought that best replicates the experimental data. Due to the limitation of the testing frame, the full hydraulic cylinder displacement cannot simply be applied as a boundary condition to the finite element test specimen. An artificial material section is therefore added to the test specimen. In this work, the central $35 \mathrm{~mm}$ of the test specimen is modelled with elastic properties as well as plastic behaviour using an MTS user subroutine in Abaqus 6.11 [1]. An additional $5 \mathrm{~mm}$ of artifical elastic material is added to both ends of the test specimen. A representation of this can be seen in Figure 2(a). The hydraulic cylinder displacement can now be used as a boundary condition while the stiffness of the artificial material section can be changed during the inverse analysis procedure. By modelling it in this way, the experimental hydraulic cylinder displacement can applied to the artificial material section. A correct choice of artificial material stiffness would then result in an effective strain rate and displacement experienced by the test specimen that best resembles the uniaxial conditions experienced during the actual test.

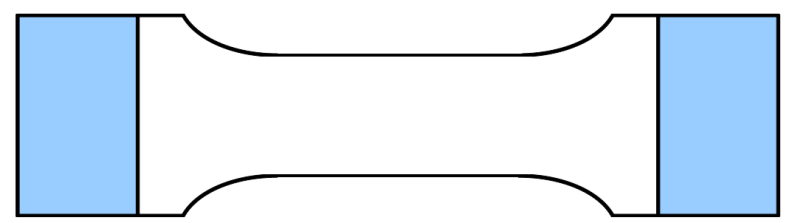

(a)

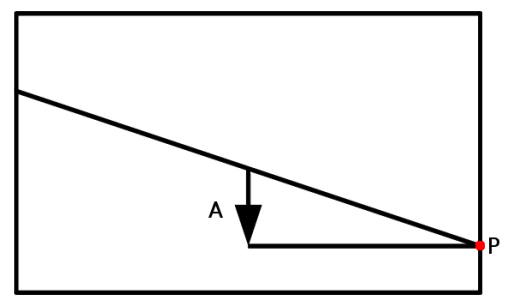

(b)

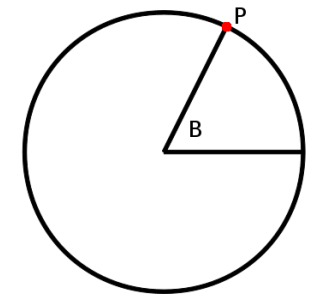

(c)

Figure 2. (a) Simple schematic illustrating the test section modelled with artificial material sections to accommodate the machine test frame stiffness. The two variables $A$ and $B$ that are used to describe the boundary displacement at a specific time are visible in (b) and (c). 
The strain gauge data visible in Figures 1(a), (b) and (c) seems to deviate early on in the experiment. From this observed difference in the strain gauge data in a single compression test, it was decided to include a spatially varying displacement boundary condition to the model instead of just a uniaxial displacement. If the uniaxial direction is chosen as the $z$-axis in the finite element analysis, a top face node $n$ experiences a $z$-displacement of

$$
z_{n}=t\left(Z_{h c}+A\left(\sin (B) y_{n}+\cos (B) x_{n}\right)\right)
$$

where $t$ is the current fraction of time, $t \in[0,1]$ and $Z_{h c}$ is the final hydraulic cylinder displacement at $t=1 . x_{n}$ and $y_{n}$ are the $x$ and $y$ coordinates of node $n$ with $A$ and $B$ two boundary variables as illustrated in Figures 2(b) and (c). The $z$-displacement field at a bottom face node is determined by

$$
z_{n}=-A t\left(\sin (B) y_{n}+\cos (B) x_{n}\right) .
$$

This is a simple linearly varying displacement boundary condition that also increases linearly over time.

From the data no constant thermal stress component $\sigma_{i}$ is assumed. The constant thermal stress component of the mechanical threshold stress definition in Equation (5) therefore falls away. Neither $k$ nor $b$ are used anywhere other than the relationships ${ }^{k} / g_{o \varepsilon} b^{3}$ and ${ }^{k} / g_{o \varepsilon s} b^{3}$, so these two relationships are used as single variables. A list of the remaining MTS material parameters used as optimisation variables and constant parameters are given in Table 1.

Table 1. List of MTS material parameters regarded as either constant or optimisation variables for use in the inverse analysis.

\begin{tabular}{lrrr}
\hline Parameter & Definition & Type & Value \\
\hline$\sigma_{a}$ & Equation 1 & Variable & \\
$\hat{\sigma}_{\varepsilon s o}$ & Equation 9 & Variable & \\
$k / g_{o \varepsilon} b^{3}$ & Equation 6 & Variable & \\
$k / g_{o \varepsilon s} b^{3}$ & Equation 9 & Variable & \\
$\theta_{o}$ & Equation 8 & Variable & \\
$\mu_{o}$ & Equation 2 & Variable & \\
$D_{o}$ & Equation 2 & Variable & \\
$\hat{\sigma}_{\varepsilon}$ & Equation 5 & Constant & 0 \\
$T_{o}$ & Equation 2 & Constant & 200 \\
$\dot{\varepsilon}_{o}$ & Equation 4 & Constant & $10^{6}$ \\
$\dot{\varepsilon}_{\varepsilon s o}$ & Equation 9 & Constant & $10^{6}$ \\
$q_{\varepsilon}$ & Equation 6 & Constant & 1 \\
$p_{\varepsilon}$ & Equation 6 & Constant & $2 / 3$ \\
$\alpha$ & Equation 8 & Constant & 2 \\
\hline
\end{tabular}


The seven MTS material parameters are determined along with an artificial material stiffness at $25^{\circ} \mathrm{C}$ and $500^{\circ} \mathrm{C}$ respectively. Including the two boundary condition variables $A$ and $B$, totalling 11 variables that needs to be determined by the inverse analysis. The optimisation is performed using the unconstrained optimisation algorithm fmin, available via the scipy . optimize [8] module in Python [7].

The objective function used in the inverse analysis optimisation procedure compares the results of a finite element simulation to experimental curves in a mean squared fashion. Given a set of parameter values, a room temperature analysis is set up which includes the displacement field boundary conditions of Equations (10) and (11), as well as the artificial material stiffness. This problem is then solved using Abaqus [1].

The logarithmic strains at the three locations spread evenly around the centre circumference of the finite element test specimen is then compared to the strain gauge data as a function of time. The load cell data is also compared to the total vertical reaction force at the top face. The $773 \mathrm{~K}$ experiment is assumed to have occured under ideal circumstances, since only a single set of strain data is available for comparison. A quarter model of the schematic visible in Figure 2(a) is used. The same uniaxial displacement over time is used as extracted from the hydraulic cylinder displacement data for the $773 \mathrm{~K}$ simulation. The average logarithmic strain of the central element set and reaction force at the top face is then compared to the strain and load cell data for the corresponding experiment. This was done on the material test for the room temperature results depicted in Figures 1(a) and (d). The converged results are displayed in Figures 3 and 4 respectively.

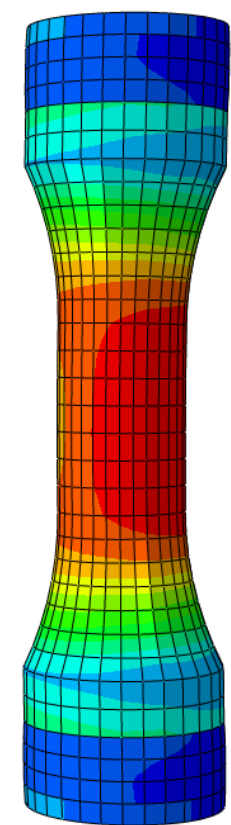

(a)

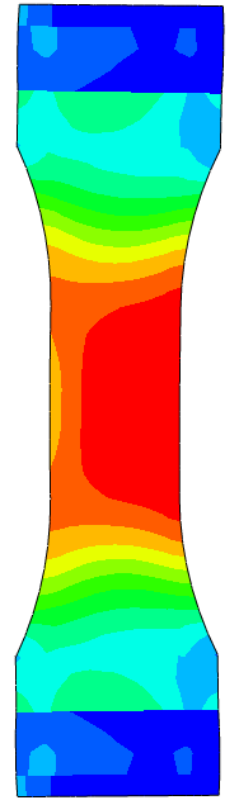

(b)

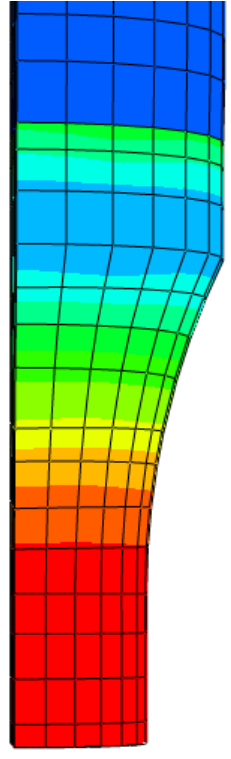

(c)

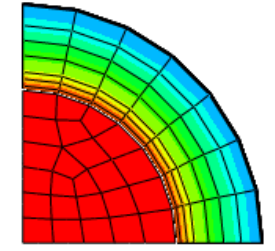

(d)

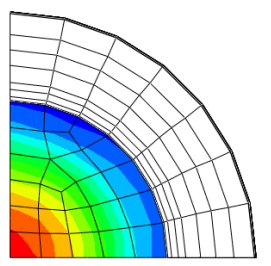

(e)

Figure 3. FEA results showing the von Mises stresses at the end of the room temperature simulation where a bend is included in the model in (a) and (b). (c) The upper part of the uniaxial compression quarter model for $500^{\circ} \mathrm{C}$. A detail of the von Mises stress in a uniaxial compression simulation is visible in (d) with contours scaled so that the gradients can be seen in (e). 


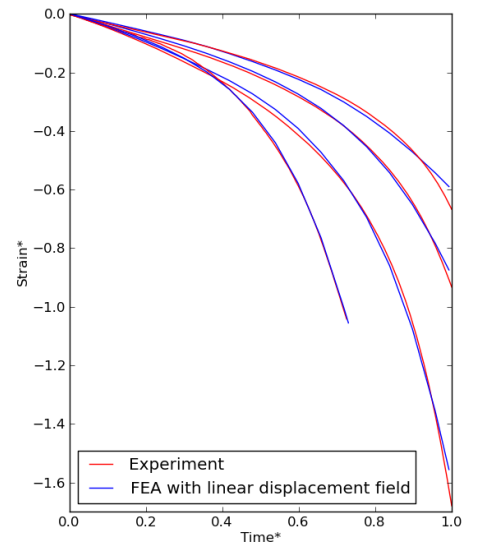

(a)

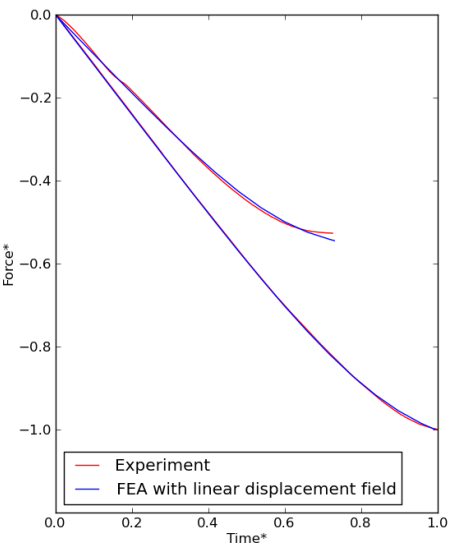

(b)

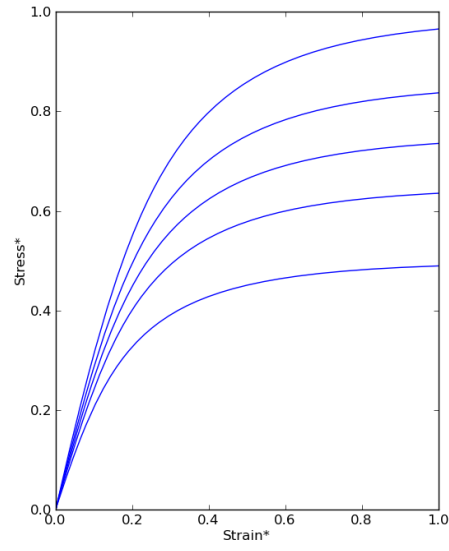

(c)

Figure 4. Resulting optimum fit of the FEA curves compared to the experimental data. (a) The three strain gauge measurements as well as the extensometer data compared to what the finite element model predicts. (b) FEA and experimental forces over time. (c) The MTS material response at $25^{\circ} \mathrm{C}, 150^{\circ} \mathrm{C}, 250^{\circ} \mathrm{C}, 350^{\circ} \mathrm{C}$ and $500^{\circ} \mathrm{C}$ for a constant strain rate of $0.0001 \mathrm{~s}^{-1}$.

In Figures 3(a) and (b) as well as the actual strains over time in 4(a), it is visible that a very small equivalent bend is necessary to approximately replicate the distribution of strains around the central circumference of the test section. The five curve fits in 4(a) and (b) match well but could be improved. The mismatch is most likely due to inadequate parameterisation of the boundary conditions. This parameter identification required 1'156 function evaluations to converge. Each function evaluation required approximately $45 \mathrm{~s}$, making the inverse parameter identification a very expensive exercise if done using the finite element procedure. It is assumed that a proper parameterisation of the boundary conditions would improve the material parameter estimation, however, the proper parameterisation is also unknown. A more complex boundary condition parameterisation would also require additional unknowns which could result in an even more computationally expensive procedure.

\subsection{Point Integration}

Instead of using a finite element analysis where boundary conditions are constructed from various unknowns, a far cheaper choice would be to determine material properties with the help of something like a single point integration procedure. A Mechanical Threshold Stress routine is written in Python to perform the approximate parameter identification. This routine takes strain history as a function of time and determines the equivalent force. The force at each data point is determined by multiplying the yield stress with an appropriate area. Figure 5 shows the effect of using the initial area, as well as the effect of a proper poisson area compensation. A uniaxial Abaqus simulation is used as a reference. The force versus strain for the Abaqus simulation is given as the red lines of Figure 5. The same MTS material parameters as used in the FEA simulation are then used in the single point integration method.

In the one case, the force is determined by

$$
F(t)=\frac{1}{4} \pi D_{o}^{2} \sigma_{y}(t)
$$




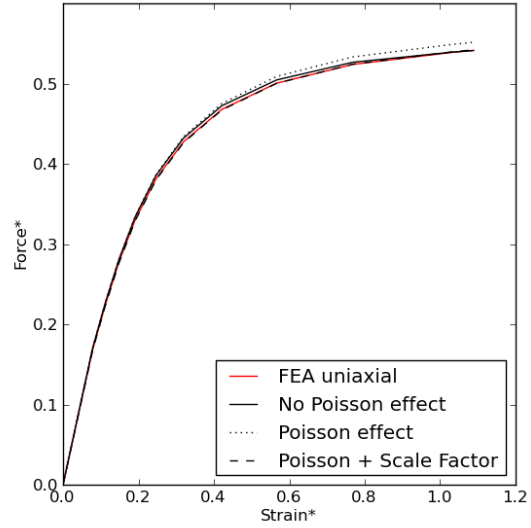

(a)

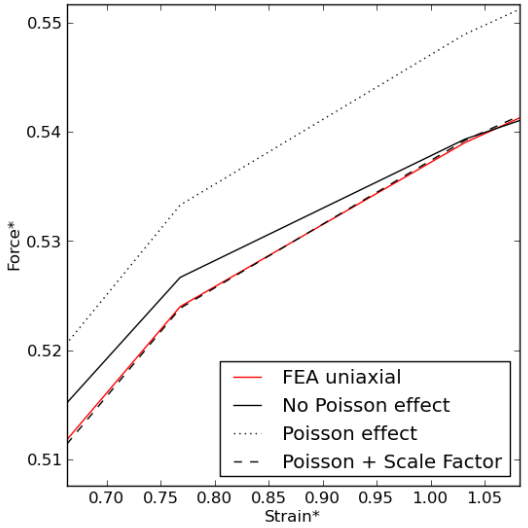

(b)

Figure 5. Normalised force versus strain curves. Uniaxial compression FEA curve compared to a single point integration curve where initial area is used, proper area compensation is applied and combined with a 0.99 scale factor. A full curve is visible in (a) with detail in (b).

with $D_{o}$ the initial test section diameter of $7 \mathrm{~mm}$. A proper area compensation, with a scale factor is seen to have the best fit to the reference curve. This scale factor is included and is motivated by the stress gradients seen in Figure 3(e). The chosen compensation for area and resulting force calculation is determined from

$$
F(t)=\frac{S_{f}}{4} \pi\left[D_{o}\left(1+\nu \varepsilon_{e}(t)+0.5 \varepsilon_{p}(t)\right)\right]^{2} \sigma_{y}(t)
$$

where $\varepsilon_{e}(t)$ is the elastic strain component at a given time and $\varepsilon_{p}(t)$ is the plastic strain component. The proper area compensation takes elastic and plastic strains into account, while a scale factor $S_{f}$ of 0.99 was chosen. This area compensation scale factor is seen to produce a fairly good match to the finite element uniaxial simulation of Figure 5.

One way of using the room temperature test to approximate the MTS material parameters, is by taking the average of the three strain gauge readings and assuming that this is the effective strain rate for a single element. Another approach involves the approximation of the room temperature test by three distinct cylindrical elements, each with one third the area of the actual specimen. In the latter approximation, the three strain gauge readings are used to construct a linear varying strain field. This is done by using the coordinates of the strain gauges in their original locations seen in Figure 6 to construct a spatially varying strain field of the form

$$
\varepsilon(x, y, t)=C_{0}(t)+C_{1}(t) x+C_{2}(t) y
$$

The time dependent equivalent strains at the centroids of the $120^{\circ}$ sectors are then determined by inserting the centroid coordinates into Equation (14). The resulting strains as a function of time are used to determine each of the three cylindrical elements' contribution to the force in the same way as the single cylinder approximation, using only a third of the full specimen area. The single cylinder (average strain) procedure and three cylinder procedures are now compared and evaluated using virtual problems. 


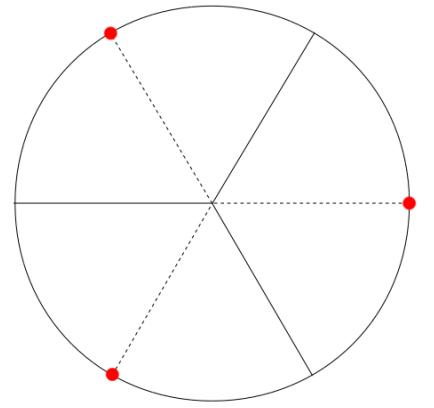

(a)

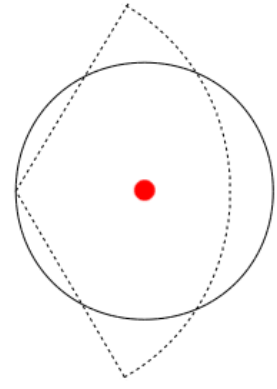

(b)

Figure 6. (a) The location of the three strain gauges equally spaced around the circumference of the central test section. During the three cylinder approximation, a $120^{\circ}$ circular sector is modelled by a cylindrical element with the same area. (b) The effective cylindrical approximation to a $120^{\circ}$ sector with the centroids coinciding.

In the first virtual problem, a finite element analysis is run with a linear displacement field boundary condition similar to the one described in Equations (10) and (11). The strains at the three locations around the central circumference and the reaction force is extracted from the finite element simulation and treated as if it were experimental data. A parameter identification is then performed where the seven MTS material parameters are determined using numerical optimisation. In the single cylinder procedure, the average of the three strain gauges as a function of time is used in to determine the forces for a given set of material parameters. In the three cylinder procedure, a linear interpolation of the strains is used at each sector centroid location and the smaller equivalent cylinder contribution to the total force is used to determine the force as a function of time. The squared difference between the approximated and virtual experiment forces for each time increment is used as the current function value. The optimisation is again performed using the fmin algorithm.

The converged normalised results are visible in Table 2 . The converged curve fit of the normalised forces as a function of time is visible in Figure 7(c). The force over strain is also plotted in Figure 7(a) using the average of the tree strains for the single cylinder approximation and Figure 7(b) using the virtual experimental strains and three cylinder approximation.

Table 2. The single cylinder and three cylinder approximation to the material parameters, given the first set of vitual experimental data.

\begin{tabular}{lrrr}
\hline Parameter & Exact value & Single cylinder procedure & Three cylinder procedure \\
\hline$\sigma_{a}$ & 1.0000 & 1.00771882 & 1.00547183 \\
$\hat{\sigma}_{\varepsilon s o}$ & 1.0000 & 0.94674572 & 0.97238436 \\
$k / g_{o \varepsilon} b^{3}$ & 1.0000 & 0.97057691 & 0.96244947 \\
$k / g_{o s s} b^{3}$ & 1.0000 & 1.62117934 & 1.36908000 \\
$\theta_{o}$ & 1.0000 & 1.02377007 & 1.04064404 \\
$\mu_{o}$ & 1.0000 & 1.00701314 & 1.00479798 \\
$D_{o}$ & 1.0000 & 1.00450078 & 0.99620249 \\
\hline
\end{tabular}




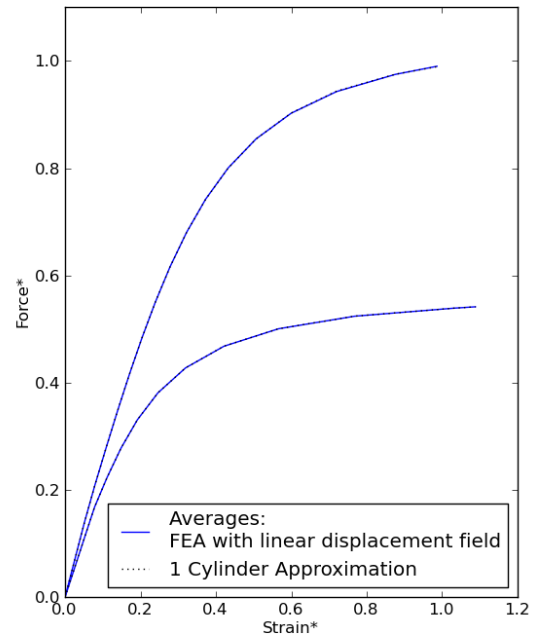

(a)

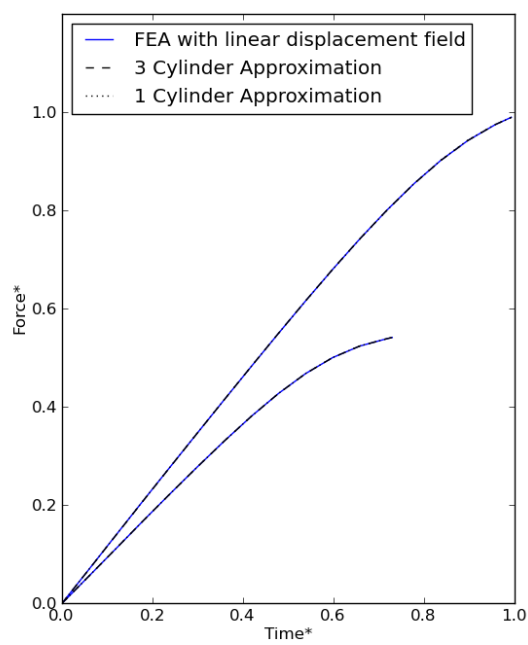

(c)

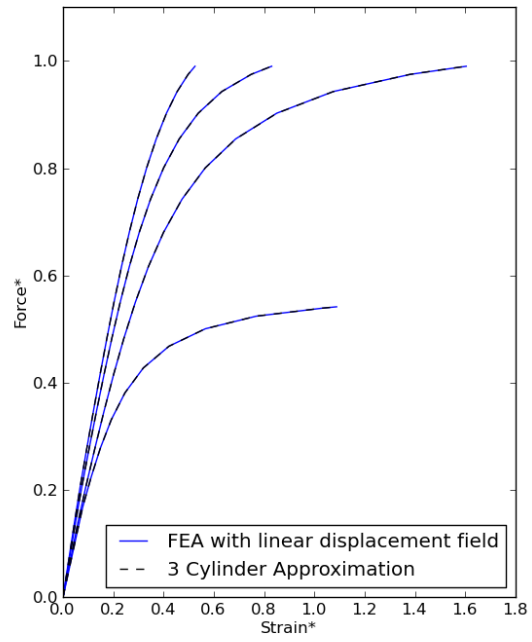

(b)

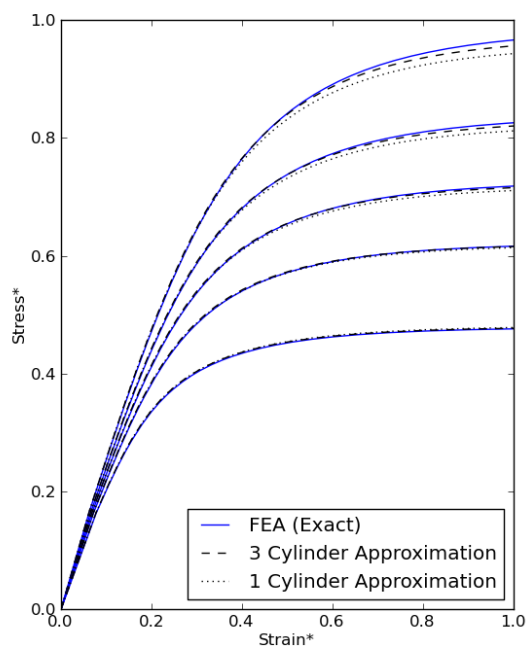

(d)

Figure 7. Comparison of the single cylinder and three cylinder procedures to fit MTS material parameters for a linear displacement distribution. (a) Normalised force versus strain using a single cylinder and the average of the three strains in the room temperature comparison. (b) Normalised force versus strain using a three cylinder fit and the three finite element strains. (c) Normalised force as a function of time fitted using the single and three cylinder approximation. (d) The known true MTS material response verus that determined from the single and three cylinder procedures for a constant strain rate of $0.0001 \mathrm{~s}^{-1}$. 
Table 3. The single cylinder and three cylinder approximation to the material parameters, given the second set of vitual experimental data.

\begin{tabular}{lrrr}
\hline Parameter & Exact value & Single cylinder procedure & Three cylinder procedure \\
\hline$\sigma_{a}$ & 1.0000 & 1.51329714 & 1.06111960 \\
$\hat{\sigma}_{\varepsilon s o}$ & 1.0000 & 0.87864129 & 0.96890237 \\
$k / g_{o \varepsilon} b^{3}$ & 1.0000 & 1.10881247 & 1.22509147 \\
$k / g_{o \varepsilon s} b^{3}$ & 1.0000 & 0.71519620 & 0.62102419 \\
$\theta_{o}$ & 1.0000 & 0.45646766 & 0.79337991 \\
$\mu_{o}$ & 1.0000 & 1.01079021 & 1.03017389 \\
$D_{o}$ & 1.0000 & 1.14494185 & 1.10360844 \\
\hline
\end{tabular}

The material response is determined given the converged MTS material parameters. The constant strain rate curves at a strain rate of $0.0001 \mathrm{~s}^{-1}$ is plotted for $25^{\circ} \mathrm{C}, 150^{\circ} \mathrm{C}, 250^{\circ} \mathrm{C}$, $350^{\circ} \mathrm{C}$ and $500^{\circ} \mathrm{C}$. Comparing these curves to the response using the known virtual experiment parameters used in the FEA simulation, it appears that both procedures are able to approximate the actual material with reasonable accuracy. This should therefore also hold true for the experimental data if the experiment was subject to sufficiently small strains. Although neither procedure replicates the known response exactly, the three cylinder procedure performs the best.

A second virtual experiment is run with the exact same MTS material parameters. A more complex boundary condition is applied so that the angle and magnitude of the displacement field reference point have a nonlinear time dependance. The force and three strains obtained from a finite element analysis is again used as virtual experimental data.

The converged single cylinder and three cylinder approximation results are visible in Table 3 for the second virtual experiment. The associated curve fits are visible in Figure 8. The material response using the converged parameters are compared to the actual material in Figure 8(d). Since this problem has a more complex and higher strain, the single and three cylinder procedures do not approximate the true material response as well as in the first virtual experiment. In this example, the three cylinder approximation does noticeably better than the single cylinder equivalent, but it is evident that the three strains should be properly coupled as it would be in a finite element analysis.

If the real experimental tests are closer related to the first virtual problem with smaller strains, it is likely that the one and three cylinder procedures would be well suited to determine the material parameters which would closely approximate the actual material response. These two methods are now used on the actual experimental data.

\subsection{Results on Actual Experimental Data}

The MTS material parameters of an experimentally tested material is now determined using the one and three cylinder procedures. This is again done on the material test of which the room temperature data is represented in Figure 1(a) and (d). The strain history and its associated time data is used the same way as mentioned in the virtual experiments to obtain the force curve as a function of time. The converged curve fit are displayed in Figure 9.

In this case, both the single cylinder and three cylinder procedure returned material 


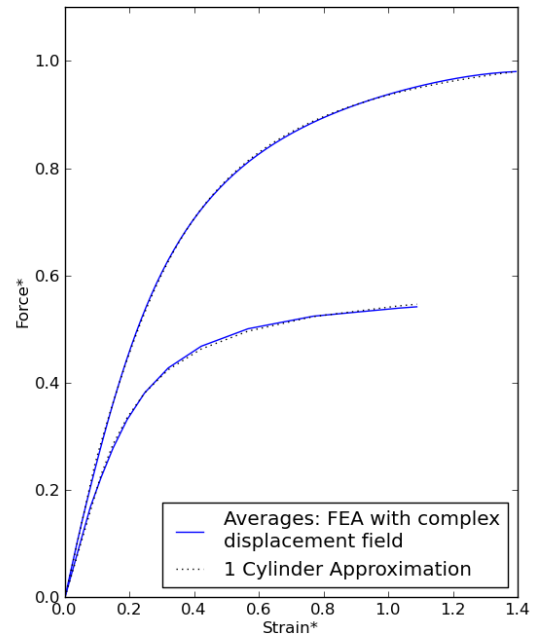

(a)

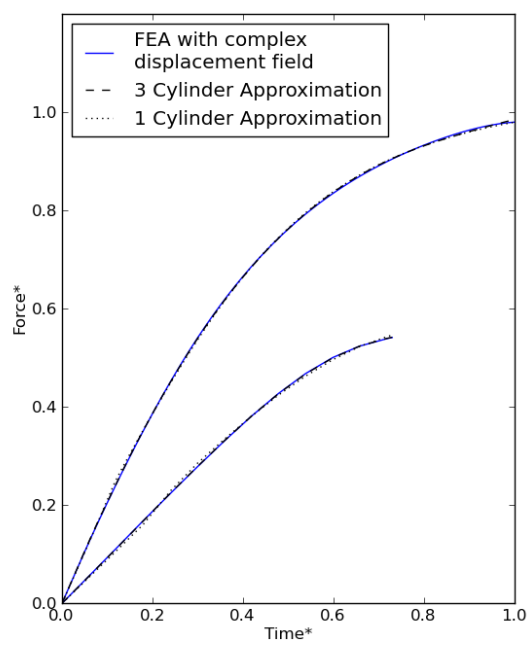

(c)

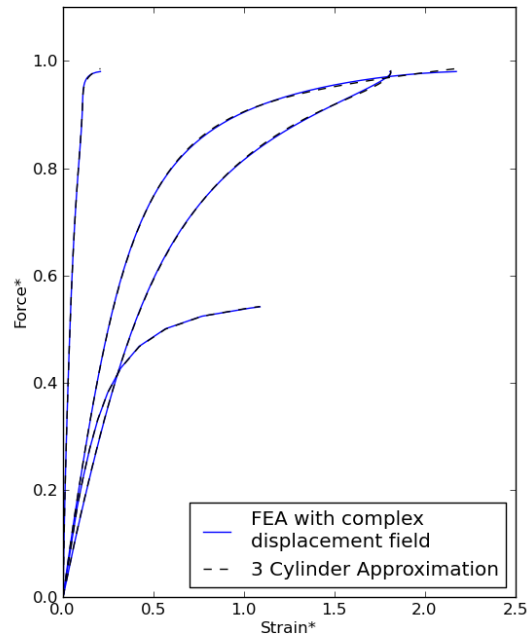

(b)

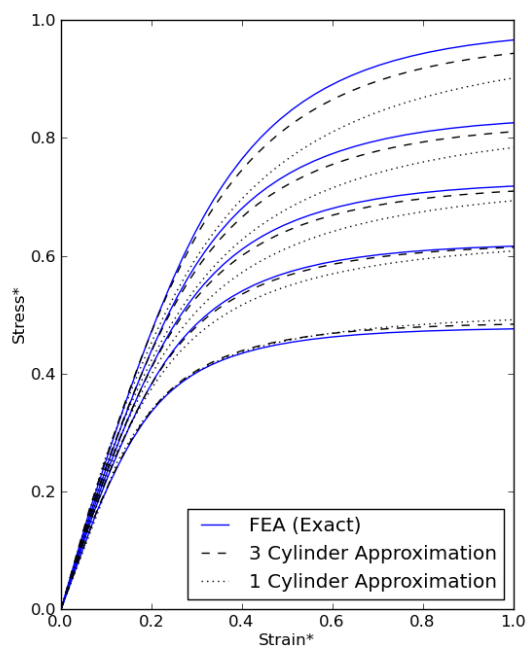

(d)

Figure 8. Comparison of the single cylinder and three cylinder procedures to fit MTS material parameters for an arbitrary selected complex displacement distribution. (a) Normalised force versus strain using a single cylinder and the average of the three strains in the room temperature comparison. (b) Normalised force versus strain using a three cylinder fit and the three finite element strains. (c) Normalised force as a function of time fitted using the single and three cylinder approximation. (d) The known true MTS material response verus that determined from the single and three cylinder procedures for a constant strain rate of $0.0001 \mathrm{~s}^{-1}$. 


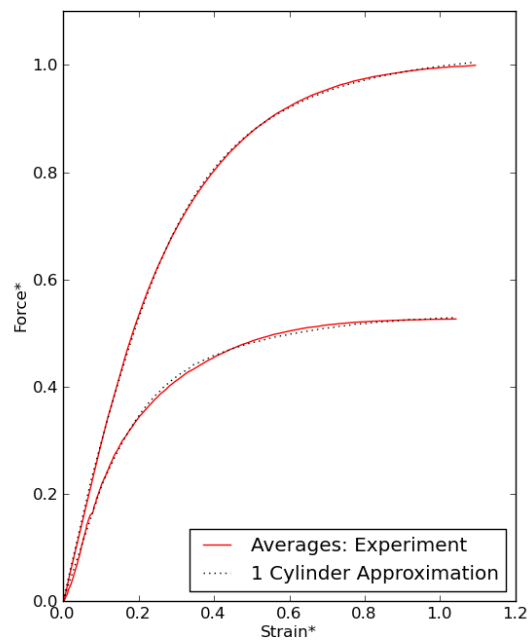

(a)

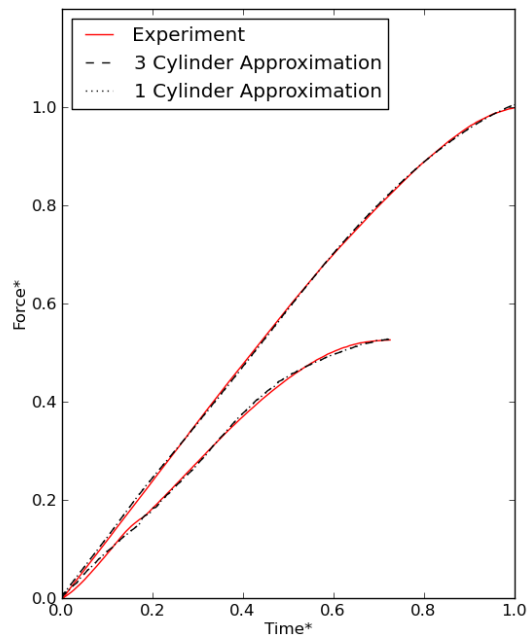

(c)

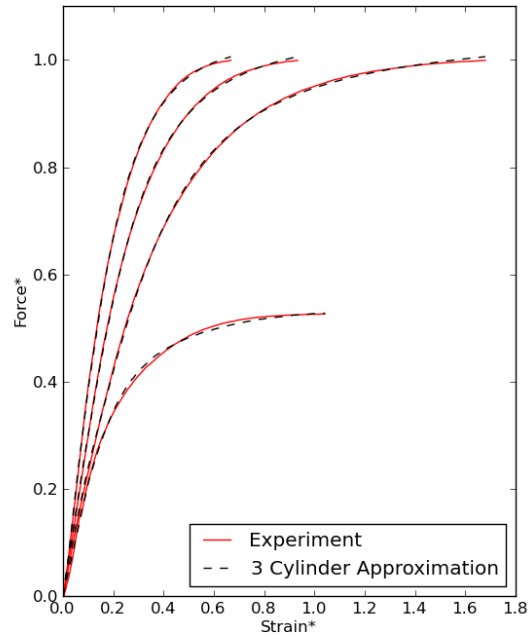

(b)

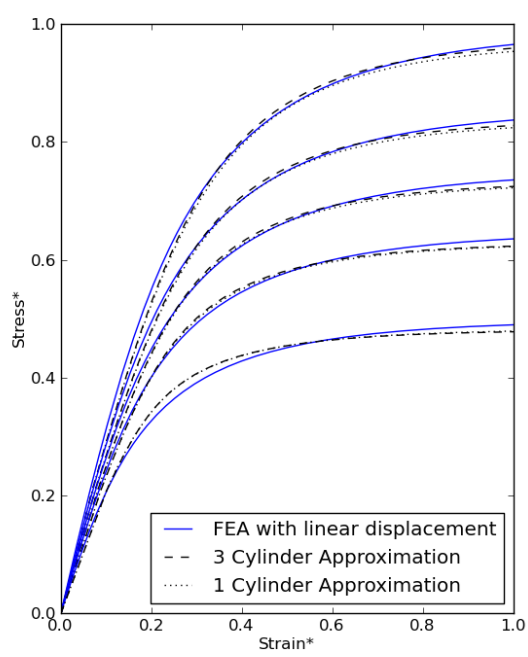

(d)

Figure 9. Comparison of the single cylinder and three cylinder procedures to fit MTS material parameters on experimental data compared to the MTS material determined from the inverse finite element analysis. (a) Normalised force versus strain using a single cylinder and the average of the three strain gauges in the room temperature comparison. (b) Normalised force versus strain using a three cylinder fit and the three gauge strains. (c) Normalised force as a function of time fitted using the single and three cylinder approximation. (d) The inverse FEA determined MTS material response verus that determined from the single and three cylinder procedures for a constant strain rate of $0.0001 \mathrm{~s}^{-1}$. 
parameter values that result in similar material response. The materials determined by the single and three cylinder procedures also closely match the material response using the parameters determined by the finite element inverse analysis of which the results could be seen in Figure 4.

From Figure 9(d), the exact material response is not clear but it is evident that all of the methods result in similar material response required to approximate the experimental results. Comparing the results of Figure 7(d) and 9(d), it is also possible that the one and three cylinder approximated materials could come close to the true material response. A better boundary parameterisation should be investigated if an increased accuracy and certainty in the material response is required. Ideally, the full finite element inverse analysis should allow for improved modelling of the experiment in order to accurately determine the material parameters.

Considering the results presented in this conference contribution, further investigation will be given to accurately determine the material parameters and boundary conditions that approximate the experimental data with a full finite element inverse analysis. This future investigation should mainly find a proper displacement boundary parameterisation so that the experimental data can be better approximated.

\section{REFERENCES}

[1] Dassault Systèmes., "Abaqus FEA software", 6.11., SIMULIA web site, $\mathrm{URL}=$ http://www.3ds.com/products/simulia/portfolio/

[2] Chen, S. R., Gray, G. T., "Constitutive Behaviour of Tantalum and Tantalum-Tungsten Alloys.", Metall. and Mat. Trans. 27(A), 2994-3006, 1996.

[3] Dunlay, W. A., Tracy, C. A., Perrone, P. J., 1989, "A Proposed Uniaxial Compression Test for High Strength Ceramics.", Report: U.S. Army Materials Technology Laboratory MTL-TR-89-89.

[4] Follansbee, P. S., Kocks, U. F., "A Constitutive Description of Copper Based on the Use of the Mechanical Threshold Stress as an Internal State Variable.", Acta Metall. 36, 81-93, 1988.

[5] Kocks, U.F., Argon, A.S., Ashby, M.F., “Thermodynamics and Kinetics of Slip.”, In: Progress in Materials Science 19, Pergamon Press, New York. 1975.

[6] Kocks, U.F., Tomé, C.N., Wenk, H.-R., “Texture and Anisotropy.”, Cambridge University Press, 1998.

[7] Python programming language., 2.6., URL = www. python.org

[8] SciPy: Scientific Tools for Python., 0.8.0., URL = www.scipy.org

[9] Varshni, Y.P., "Temperature Dependence of the Elastic Constants.", Phys. Rev. B 2, 3952-3958. 1970. 